\title{
Cardiac actinomycosis in a patient presenting with acute cardiac tamponade and a mass mimicking pericardial tumour
}

\author{
L Jánoskuti, M Lengyel, T Fenyvesi
}

Heart 2004;90:e27 (http://www.heartinl.com/cgi/content/full/90/5/27). doi: 10.1136/hrt.2003.031633

\begin{abstract}
A case of pericardial actinomycosis mimicking a pericardial tumour is reported. After the appearance of non-specific subpleural pulmonary nodules, a 48 year old woman presented with fever and clinical signs of pericardial tamponade. Subxiphoid pericardiotomy yielded a culture negative fluid and inflammatory reactive histopathology in the pericardial biopsy specimen. Because of suspected infection cefamandole was administered for 10 days and the patient became afebrile. The pericardial effusion recurred with no clinical signs two weeks later. Steroid medication resulted in rapid regression of the pericardial effusion. Subsequent echocardiography controls showed a tumour-like pericardial mass, confirmed by cardiac magnetic imaging. Surgical exploration led to the final histological diagnosis of actinomycosis. After high dose and long term penicillin $G$ treatment the patient recovered fully with no recurrence during two years' follow up.
\end{abstract}

C ardiac actinomycosis is a rare disease. Pericardial involvement in actinomycosis was discussed in two comprehensive reviews ${ }^{12}$ and only a few case reports have been published. ${ }^{3-7}$ Pericardial involvement has been characterised by pericardial effusion that evolves into cardiac tamponade or constrictive pericarditis. We report a case of pericardial actinomycosis mimicking a pericardial tumour.

\section{CASE REPORT}

A 48 year old woman presented to our outpatient clinic in June 1999 because of moderate dyspnoea and left sided chest pain on deep inspiration. There was nothing peculiar in her history. She had been a moderate smoker (3-4 cigarettes a day) and consumed about one alcoholic drink a day. Physical and routine laboratory findings did not show any abnormality. On chest radiography we noted normal heart contours, a small, not well delineated shadow in the lateral part of the left middle lung field, and a small left sinus pleural effusion. Chest computed tomography showed multiple subpleural nodules of 3-5 $\mathrm{mm}$ and a larger one consistent with the radiographic finding.

We planned further investigation but the patient returned to our clinic after only four weeks with recent onset retrosternal pain, fever, and clinical signs of pericardial tamponade. Laboratory results were white cell count $10.1 \mathrm{~g} / \mathrm{l}$, packed cell volume 0.34 , haemoglobin $110 \mathrm{~g} / \mathrm{l}$, mean corpuscular volume $86 \mathrm{fl}$, platelet count $343 \mathrm{~g} / \mathrm{l}$, and erythrocyte sedimentation rate $93 \mathrm{~mm} / \mathrm{h}$. Echocardiography showed characteristic signs of pericardial tamponade. Surgical subxiphoid pericardial fenestration was performed, $600 \mathrm{ml}$ of clear pericardial fluid was withdrawn, and a histological specimen was taken from the pericardium and pleura. The culture was negative and there were more polymorphonuclear leucocytes in the sediment. Histological analysis found aspecific polymorphonuclear infiltration both in the pleural and in the pericardial specimen. No signs of malignancy were seen. Because of suspected infection cefamandole was administered for 10 days and the patient became afebrile.

The pericardial effusion recurred with no clinical signs in two weeks' time. Immunology status including HIV enzyme linked immunosorbent assay (ELISA) and investigations to rule out possible malignancy including gynaecological examination, abdominal ultrasound and computed tomography, gastroscopy, and colonoscopy were negative. The pericarditis of unknown origin prompted us to start steroid medication (methylprednisolone) resulting in rapid regression of the pericardial effusion. Subsequent echocardiography controls, however, showed a gradual thickening of the pericardium.

On 7 September a mass $3 \times 3 \mathrm{~cm}$ suspected to be a tumour at the right ventricular apex was noted. The right ventricular wall appeared immobile and seemed to be infiltrated by the mass. Cardiac magnetic resonance imaging (MRI) was performed (10 September 1999), which showed an extracardiac mass in front of the anteroseptal groove on the epicardial surface of the pericardium with uneven contours (fig 1). The patient's general condition worsened. Fever and purulent pleural effusion appeared. The fluid was culture negative with a very high concentration of polymorphonuclear leucocytes but there were no other diagnostic findings on Gram stain. Operative exploration was decided upon. On 13 October 1999 a left sided axillary thoracotomy was performed. Multiple rice sized greyish nodules were seen on the pleural and pericardial surface. A mass $3 \times 4 \mathrm{~cm}$ was observed in the pericardium at the right ventricular apex. Histopathological analysis disclosed colonies of Actinomyces israelii (fig 2). Twenty million units a day of intravenous penicillin $G$ was started immediately and was given for five weeks. Subsequently the patient was given $6 \mathrm{MU} /$ day of oral penicillin until 27 June 2000. The patient recovered fully. Laboratory measurements and sedimentation rate were normal. Repeat echocardiography and cardiac MRI showed that the pericardial mass had almost disappeared. No recurrence of the disease was detected during two year's follow up till her recent death in an accident.

\section{DISCUSSION}

In a most detailed analysis by Cornell and Shookhoff ${ }^{1}$ presumably all 68 cases of cardiac actinomycosis published by 1944 were summarised. Since this publication it has been suggested that the heart has been involved in only about $2 \%$ of all cases of this infection, with the pericardium as the most common site. The usual presentation of pericardial actinomycosis is pericardial effusion that may evolve into cardiac tamponade or constrictive pericarditis. ${ }^{1-7}$ Diagnosis of 


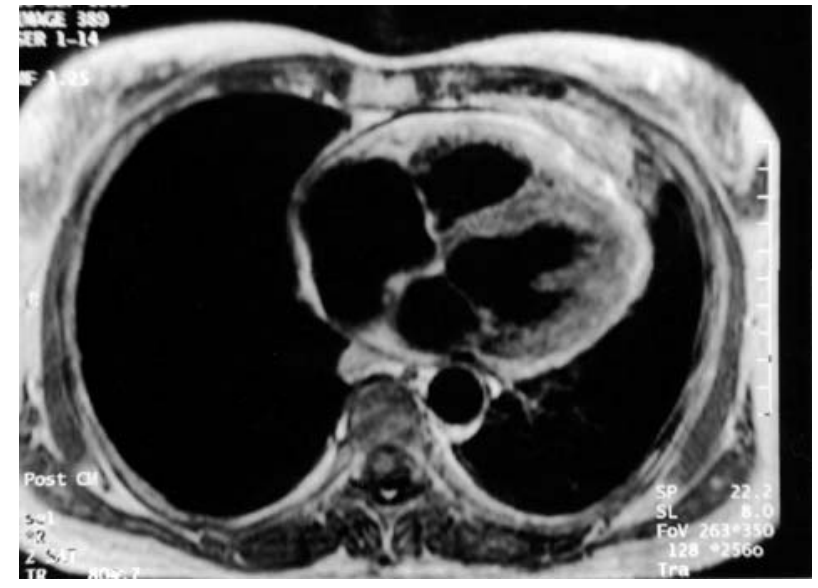

Figure 1 ECG gated saturation pulse prepared $\mathrm{Tl}$ weighted sequence (dark blood technique) in the coronal cardiac plane was used to generate this image. The image shows that an extracardiac mass with uneven contours is placed exactly in front of the anteroseptal groove on the epicardial surface of the pericardium.

actinomycosis is generally hampered by the difficulty in isolation and culture of the organism. It must be cultured in strictly anaerobic conditions. In the review of Fife and colleagues, ${ }^{2}$ purulent pericardial fluid was obtained from 10 (53\%) of 19 patients. However, A israelii was successfully cultured from the fluid in only two cases. In the majority of cases in the literature actinomycosis has been diagnosed by histopathology. The so called sulphur granules of actinomycosis feature surface clubs visible by light microscopy. In the case reported by Shinagawa and colleagues, ${ }^{7}$ the pericardial biopsy failed to provide convincing evidence for pericardial actinomycosis but culture of the pericardial effusion gave a positive growth of $A$ israelii. Pulmonary or anterior mediastinal masses with pericardial effusion have been reported in the literature ${ }^{356}$ but to our knowledge our case is the first where pericardial actinomycosis mimicked a pericardial tumour.

In conclusion, we described a case of pericardial actinomycosis. After the appearance of non-specific subpleural pulmonary nodules the patient presented with symptoms of acute pericardial tamponade. Pericardial fluid culture and biopsy did not lead to a diagnosis of actinomycosis. Echocardiography showed a tumour-like pericardial mass confirmed by MRI. Surgical exploration led in the final histological diagnosis of actinomycosis. After high dose and long term penicillin treatment, the patient recovered fully with no recurrence during two years' follow up.

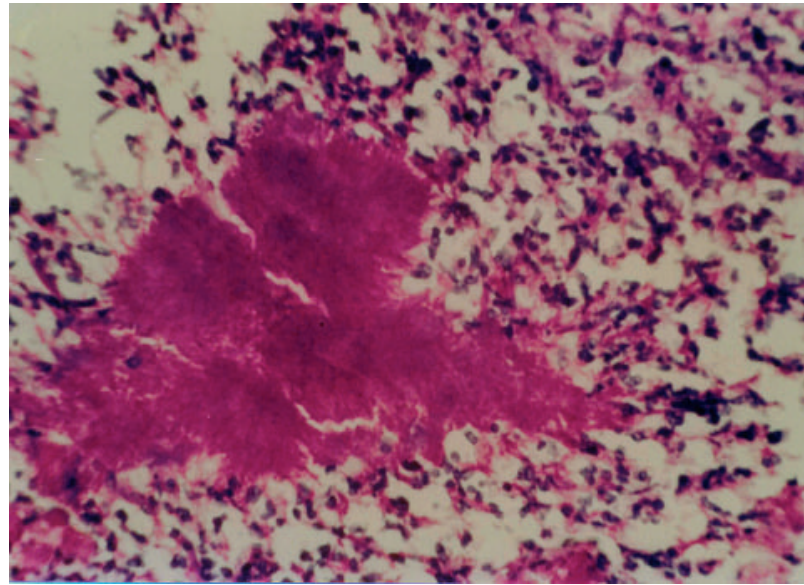

Figure 2 High power view showing the central colony of Actinomyces israelii and surrounding polymorphonuclear reactions (periodic acid Schiff stain).

\section{ACKNOWLEDGEMENTS}

The authors thank Dr I Soltész for the pathological diagnosis and Dr T Simor for his assistance in the interpretation of the cardiac MRI.

\section{Authors' affiliations}

L Jánoskuti, T Fenyvesi, III Department of Internal Medicine, Semmelweis University, Budapest, Hungary

M Lengyel, Hungarian Institute of Cardiology, Budapest, Hungary

Correspondence to: Dr L Jánoskuti, III Department of Internal Medicine, Semmelweis University, Budapest, Hungary; ismi@mail.datanet.hu

Accepted 22 January 2004

\section{REFERENCES}

1 Cornell A, Shookhoff HB. Actinomycosis of the heart simulating rheumatic fever: report of three cases of cardiac actinomycosis, with a review of the literature. Arch Intern Med 1944;74:11-27.

2 Fife TD, Finegold SM, Grennan T. Pericardial actinomycosis: case report and review. Rev Infect Dis 1991;13:120-6.

3 Garini G, Brodi C, Mazzi A, et al. Thoracic actinomycosis with lung, mediastinal and pericardial involvement: a case report. Recenti Prog Med 1995;86:107-11

4 Litwin KA, Jadbabaie F, Villanueva M. Case of pleuropericardial disease caused by Actinomyces odontolyticus that resulted in cardiac tamponade. Clin Infect Dis 1999;29:219-20.

5 Sandoval V, Orea TA, Garcia ML, et al. Chronic cardiac actinomycosis: case report and review of the literature. Arch Inst Cardiol Mex 1988;58:243-5.

6 Slutzker AD, Claypool WD. Pericardial actinomycosis with cardiac tamponade from a contiguous thoracic lesion. Thorax 1989:44:442-3.

7 Shinagawa N, Yamaguchi E, Takahashi T, et al. Pulmonary actinomycosis followed by pericarditis and intractable pleuritis. Intern Med 2002;41:319-22. 